\title{
Stabilization of invariant sets for nonlinear non-affine systems ${ }^{\text {in }}$
}

\author{
Anton S. Shiriaeve, ${ }^{\mathrm{a}, \mathrm{b}, *}$, Alexander L. Fradkov ${ }^{\mathrm{a}}$ \\ anstitute for Problems of Mechanical Engineering, 61, Bolshoy V.O., St.Petersburg, 199178 Russia \\ ${ }^{\mathrm{b}}$ The Maersk Mc-Kinney Moller Institute for Production Technology Odense University, Campusvej 55, DK-5230 Odense M, Denmark
}

Received 14 July 1998; revised 23 May 1999; received in final form 21 March 2000

\begin{abstract}
The problem of the global and local stabilization of invariant sets for general nonlinear controlled systems is considered. New state feedback stabilizing controllers and sufficient conditions of asymptotic stability of a goal set with the specified region of attraction are proposed. The proofs of the obtained results are based on the detailed analysis of the $\omega$-limit sets of the closed-loop system with the speed-gradient (or "Jurdjevic-Quinn") controllers for "affinized" controlled system. Two illustrating examples are given. (C) 2000 Elsevier Science Ltd. All rights reserved.
\end{abstract}

Keywords: Nonlinear control; Energy control; Speed-gradient method

\section{Introduction and problem statement}

Control of nonlinear oscillatory systems is an important problem with a variety of applications in mechanics, physics, vibrational technology, control education, etc. (Chernousko, Akulenko \& Sokolov, 1980; Åstrom \& Furuta, 1996; Bloch, Krishnaprasad, Marsden \& Sánchez de Alvarez, 1992; Wiklund, Kristenson \& Aström, 1993; Vorotnikov, 1998; Fradkov \& Pogromsky, 1998). Control of oscillatory systems requires achieving nonclassical control goals (swinging, synchronization) as well as describing and analyzing complex motions of the closed loop system. A new approach to stabilization of a desired level of oscillations for Hamiltonian systems based on the speed-gradient method (Fradkov, 1979) and energy objective functions was proposed in Fradkov, (1994, 1996), Fradkov, Guzenko, Hill and Pogromsky, (1995) and Fradkov, Makarov, Shiriaev and Tomchina,

\footnotetext{
This paper was not presented at any IFAC meeting. This paper was recommended for publication in revised from by Associate Editor Henk Nijmeijer under the direction of Editor Tamer Basar. The work was supported in part by RFBR (grant 99-01-00672), by the Russian Federal Program "Integration" (project A0151) and by the Norwegian Research Council.

* Corresponding address. The Maersk Mc-Kinney Moller Institute for Production Technology, Odense University, Campusvej 55, DK5230 Odense M, Denmark. Tel.: + 45-6550-3574, fax: + 45-6615-7697.

E-mail addresses: anton@mip.sdu.dk (A.S. Shiriaev), alf@ccs.ipme.ru (A.L. Fradkov).
}

(1997). Recently, it was extended to a class of nonlinear systems, see Shiriaev, (2000a,b). It was shown that the proposed control design methodology allows to achieve the desired energy hyper-surface with arbitrary small control intensity. However, the previous papers were mainly dealing only with nonlinear systems having affine control inputs.

In this work we consider the nonaffine controlled system

$\dot{x}(t)=f(x(t), u(t))$

with a state vector $x \in X=R^{n}$, an input function $u \in R^{m}$ and with smooth right-hand side $f$. It is assumed that the nonnegative objective function $V(x), V: X \rightarrow R^{1}$, is of the form

$V(x)=\|h(x)\|^{2}$,

where $h: X \rightarrow R^{k}$ is a given $C^{d}$-smooth vector function $(\|\cdot\|$ stands for the Euclidean norm).

The following control problem is considered: To find a state feedback controller and to determine conditions guaranteeing that for any solution $x(t)=x\left(t, x_{0}\right)$ of the closed-loop system with initial conditions $x_{0}$ from some set $\mathscr{D} \subset X$, the limit relation

$$
\lim _{t \rightarrow \infty} V(x(t))=0
$$

is valid. Below, the set $\mathscr{D}$ is chosen as either $\mathscr{D}=X$ (global achievement of control goal (3)) or $\mathscr{D}=\{x \in X$ : $V(x)<c\}$ with some positive constant $c$. It is convenient 
to introduce the auxiliary output

$y(t)=h(x(t))$.

The problem can be reformulated in the equivalent form: To find a controller and to determine conditions guaranteeing that along any solution $x=x\left(t, x_{0}\right)$ of the closed-loop system with the initial conditions $x_{0} \in \mathscr{D}$ the following relation holds:

$\lim y(t)=0$.

The goal (5) is related to asymptotic partial stabilization of system (1) with respect to the vector $y(t)$. The partial stabilization problem has a long history (see, e.g., Rumyantsev, 1970; Vorotnikov, 1993; Mahony, Mareels, Campion \& Bastin, 1997; Vorotnikov, 1998). However, the problem of nonlocal partial stabilization of sets was not previously considered for non-affine nonlinear systems. Most of the existing results are local and based on the feedback linearization approach. They do not take into account the "small control" requirement and do not cover the case of unbounded (noncompact) goal sets (both may be important for control of the oscillatory or rotatory motion). Note that the well-studied problem of stabilization of a single equilibrium point is just a special case of the above problem with $k=n, y=x$. In a number of papers (see, e.g. Byrnes, Isidori \& Willems, 1991; Lin, 1995,1996) efficient passivity-based algorithms for stabilization of an equilibrium were suggested.

In this paper control algorithms and conditions providing stabilization of desired invariant set (ensuring, in other words, the validity of the limit relation (3)) of the closed-loop system are proposed. The algorithms are based on the speed-gradient method, which is closely related to both the passivity approach and to the method known as the "Jurdjevic-Quinn approach" in Western literature. It is also shown that even for the case $k=n, y=x$ the presented results extend the results of Byrnes et al. (1991) and Lin (1995, 1996), see Remark 9 and Example 2.

Note that, the extension of existing results for point stabilization to the case of set stabilization is not obvious. It requires careful use of La Salle invariance principle since the standard zero-state detectability conditions (see e.g. Sontag, 1981; Byrnes et al., 1991; Lin, 1996) cannot be imposed (they are not adequate to the set stabilization problem). Instead, we invoke $V$-detectability conditions (Shiriaev, 2000a,b) and provide detailed analysis of the limit set for the closed-loop system. The applicability of the obtained results is illustrated by considering physical example: stabilization of given energy level of a pendulum with controlled length.

The paper is organized as follows. Section 2 contains assumptions, useful notions, and notation. The main results and discussion are given in Section 3. In Section 4, two examples are considered. The proofs of the formulated results are collected in Section 5, some conclusions are drawn in Section 6.

\section{Preliminaries and assumptions}

It follows from smoothness of the vector field $f$ and the Hadamard lemma (see Hartman, 1964, Lemma 3.1 p. 97), that the system under consideration can always be expressed in the affine-like form

$\dot{x}=f_{0}(x)+g_{0}(x) u+\sum_{j=1}^{m} u_{j}\left(R_{j}(x, u) u\right)$

where

$f_{0}(x)=f(x, 0), \quad g_{0}(x)=\frac{\partial f}{\partial u}(x, 0)$,

$R_{j}(x, u)$ are $(m \times m)$-matrices and $u_{j}, 1 \leq j \leq m$, are the components of the vector $u$. Define operator $L_{b(x)} a(x)$ where $a(x), b(x)$ are smooth functions (possibly vector functions of appropriate dimensions) as follows:

$L_{b(x)} a(x)=\frac{\partial a(x)}{\partial x} b(x)$.

We will use notation $\nabla_{x} a(x)=\partial a(x)^{\mathrm{T}} / \partial x$ for the gradient vector and omit the argument of the functions whenever it does not lead to confusion. Throughout the paper it will be assumed that

(A1) for any point $x$ of the phase space $X$ the inequality $L_{f_{0}(x)} V(x) \leq 0$ holds, i.e. the dynamics of the unforced system is stable with respect to the function $V$.

(A2) for any point $x$ belonging to the set $\left\{x \in X: L_{g_{0}} V(x)=0\right\}$ the identity $L_{f_{0}} h(x) \equiv 0$ is valid, i.e. the affine part of system (1) is lossless with respect to the set $\left\{x \in X: L_{g_{0}} V(x)=0\right\}$. In particular, it could be lossless with respect to the whole state space.

To solve the posed problem we will use the speed-gradient control algorithm (Fradkov, 1979, 1990)

$u(x(t))=-\gamma \nabla_{u} \dot{V}(x(t))$

or, more generally,

$u(x(t))=-\Psi\left(\nabla_{u} \dot{V}(x(t))\right)$.

Here $\dot{V}(x)$ is the full derivative along the solutions of (1) and $\Psi(z)$ is a vector-function forming a sharp angle with $z$, i.e. $\Psi(z)^{\mathrm{T}} z>0$ for any $z \neq 0$. To be more precise, we calculate the speed-gradient of $V$ with respect to the reduced or "affinized" system $\dot{x}=f_{0}(x)+g_{0}(x) u$.

Remark 1. The method of controller design based on the derivative of the goal function $V(x)$ is referred to as the "Jurdjevic-Quinn approach" in the Western literature. 
Note that in the original paper (Jurdjevic \& Quinn, 1978), only the case $V(x)=|x|^{2}$ was considered. More about the speed-gradient method can be found in the recent books (Fradkov \& Pogromsky, 1998; Fradkov, Miroshnik \& Nikiforov, 1999).

\section{Main results}

Introduce the distribution

$$
\begin{aligned}
S(x)=\operatorname{span}\left\{L_{g_{0}} h(x), L_{f_{0}} L_{g_{0}} h(x),\right. \\
\left.L_{f_{0}}^{2} L_{g_{0}} h(x), \ldots, L_{f_{0}}^{d-1} L_{g_{0}} h(x)\right\},
\end{aligned}
$$

where $\operatorname{span}\left\{v_{1}, v_{2}, \ldots\right\}$ is a linear space generated by columns of matrices $v_{1}, v_{2}, \ldots$, and $d$ corresponds to the highest continuous derivative of $V$. It is easy to see that for any point $x$ the dimension of linear space $S(x)$ cannot be greater than $k$. Consider the subset $P \subset X$, where the dimension of the linear space $S(x)$ is strictly less than $k$, i.e.

$P=\{x \in X: \operatorname{dim} S(x)<k\}$,

and denote the subset $\widehat{\Omega} \subset P$ as

$\widehat{\Omega}=\left\{x_{0} \in X:\right.$ the whole trajectory $x=x\left(t, x_{0}\right)$ of $(1)$

$$
\text { with } u=0 \text { lies in } P\} \text {. }
$$

Denote by $\rho(x)$ any smooth scalar function, which satisfies the inequality

$\max \left\|R_{j}(x, u)\right\| \leq \rho(x), \quad \forall x$,

$\|u\| \leq 1$

where the functions $R_{j}, j=1, \ldots, m$, are from expansion (6). Recall that a scalar nonnegative function $V(x)$ is said to be proper if for any $c \geq 0$ the set $\{x \in X: V(x) \leq c\}$ is compact.

Theorem 2. Given the controlled system (6), suppose that the assumptions (A1) and (A2) are valid, the nonnegative function $V(x)$ is proper and

$\widehat{\Omega} \subset V_{0}=\{x \in X: V(x)=0\}$.

Then along any solutions of the closed-loop system (6) with the controller

$u(x)=-\alpha(x) \Psi\left(\left[L_{g_{0}} V(x)\right]^{\mathrm{T}}\right)$,

the limit relation (3)

$\lim V(x(t))=0$

holds. Here $\Psi(z)$ is an arbitrary smooth vector function such that

$z^{\mathrm{T}} \Psi(z) \geq \psi(z)\|\Psi(z)\|^{2}>0, \quad \forall z \neq 0, \quad\|\Psi(z)\| \leq 1$, $\psi(z)$ is a smooth scalar function with $\psi(z) \geq \psi_{0}>0, \forall z$ $\alpha(x)$ is a smooth scalar function satisfying the inequality $0<\alpha(x) \leq \hat{\alpha}(x)$, where

$\hat{\alpha}(x)=\frac{\beta}{m\left(1+[\|\partial V(x) / \partial x\| \rho(x)]^{2}\right)}$,

$\beta$ is a positive constant with $0<\beta<\min \left\{\psi_{0}, m\right\}$, and the function $\rho(x)$ is from the inequality (11).

Remark 3. Theorem 2 remains also true for the case when instead of the relations (14) the function $\Psi$ satisfies the strict pseudo-gradient condition (see Fradkov, 1990)

$z^{\mathrm{T}} \Psi(z) \geq \psi(z)\|\Psi(z)\|^{\delta}>0, \quad \forall z \neq 0, \quad\|\Psi(z)\| \leq 1$,

where $\psi(z)$ is a smooth scalar function, $\psi(z) \geq \psi_{0}>0, \forall z$, and $2 \geq \delta$. Indeed, in this case

$\|\Psi(z)\|^{\delta} \geq\|\Psi(z)\|^{2}, \quad \forall z$

and inequality (14) is valid.

If the function $V$ is not proper anymore, then the assumptions of Theorem 2 are not satisfied, and one could easily derive an example of system, for which the conclusions of Theorem 2 fail. To formulate the local analog of Theorem 2, suppose that for some positive constant $c>0$ a closure of the set $V_{c}=\{x \in X: V(x)<c\}$ is compact. Denote

$P_{c}=\{x \in X: \operatorname{dim} S(x)<k\} \cap V_{c}$,

$\widehat{\Omega}_{c}=\left\{x_{0} \in X:\right.$ the whole trajectory

$$
\left.x=x\left(t, x_{0}\right) \text { of (1) with } u=0 \text { lies in } P_{c}\right\} .
$$

Theorem 4. Given the controlled system (6), suppose that assumptions (A1) and (A2) are valid, the function $V(x)$ is nonnegative, some $c>0$ the set $\bar{V}_{c}$ is compact, and

$\widehat{\Omega}_{c} \subset V_{0}=\{x \in X: V(x)=0\}$.

Then along any solution of closed-loop system (6) with the controller of the form

$u(x)=-\alpha(x) \Psi\left(\left[L_{g_{0}} V(x)\right]^{\mathrm{T}}\right)$,

and with the initial conditions $x_{0}$ from the set $\left\{x_{0} \in X^{n}: V(x)<c\right\}$ the limit relation (3) is valid. Here the functions $\Psi(z)$ and $\alpha(x)$ are defined as in Theorem 2.

Remark 5. In some examples instead of the inclusion (17) more rough and easier checkable dimensional condition

$\operatorname{dim} S(x)=k, \quad \forall x \in V_{c} \backslash V_{0}$

can be used. Indeed, condition (18) implies that the set $P, P=\{x \in X: \operatorname{dim} S(x)<k\}$, lies in the set $V_{0}$. Hence, $\hat{\Omega}_{c} \subset P \subset\{x \in X: V(x)=0\}$ and the inclusion (17) is fulfilled. 
Remark 6. It can be proved that among controllers (13) described in Theorems 2 and 4 there exists controller providing arbitrarily small value of control action, i.e. $\forall \varepsilon>0$ there exist $\left(\alpha(x), \Psi(z), \psi(z), \psi_{0}, \beta\right)$ such that state feedback (13) defined by these parameters satisfies the inequality $\|u\| \leq \varepsilon$.

Remark 7. Theorems 2 and 4 could be applied to systems, which are passive or state feedback passive with the storage function $V$ being constructed by using the total energy of the system or its other conserved quantities. One of such examples, a stabilization of desired rotational mode of the pendulum by changing the length of the rope, is treated in detail in this paper.

Also it is worth mentioning that Theorems 2 and 4 are the special important case of Theorem 3.3 (Shiriaev, 2000a). Indeed it can be shown (and this has its own value) that the inclusions (12) and (17) combined with assumptions (A1) and (A2) imply $V$-detectability of the affine part of the system (6) with the output (4), see Definition 2.2 (Shiriaev, 2000a), which leads eventually to the conclusions made in Theorems 2 and 4. So their proofs (Theorems 2 and 4) are similar to the proof of Theorem 3.3 (Shiriaev, 2000a) and omitted in this paper.

The assumptions imposed on the function $V(x)$ to be proper in Theorem 2 or the compactness of $\left\{x \in X^{n}: V(x) \leq c\right\}$ for some $c>0$ in Theorem 4 are mainly technical. In the next statement, we extend Theorems 2 and 4 to the case when neither the function $V$ is proper, nor there is some constant $c>0$, for which the set $\{x \in X: V(x) \leq c\}$ is compact. To this end, we impose additional condition guaranteeing, first, the continuability of all solutions of the closed-loop system (1), (13) to the interval $[0,+\infty)$ and, second, the existence of a nonempty $\omega$-limit set for any trajectory of the closedloop system (1), (13).

Theorem 8. Given the controlled system (1), suppose that assumptions (A1) and (A2) are valid, the function $V(x)$ is nonnegative, for some $c>0$ the functions $f(x, u), L_{g_{0}} V$, $L_{f} L_{g_{0}} V$ are bounded on the set $\Lambda=V_{c} \times\{u:\|u\| \leq 1\}$. Take the controller of the form

$u(x)=-\alpha(x) \Psi\left(\left[L_{g_{0}} V(x)\right]^{\mathrm{T}}\right)$,

where $\Psi(z)$ is an arbitrary smooth vector function such that inequality (14) holds for some smooth scalar function $\psi$ with $\psi(z) \geq \psi_{0}>0 ; \alpha(x)$ is any smooth scalar function such that $\partial \alpha(x) / \partial x$ is bounded on $\Lambda$ and that the inequality $0<\varepsilon \hat{\alpha}(x) \leq \alpha(x) \leq \hat{\alpha}(x)$ is valid for some $0<\varepsilon<1$, where

$\hat{\alpha}(x)=\frac{\beta}{m\left(1+[\|\partial V(x) / \partial x\| \rho(x)]^{2}\right)} ;$

$\beta$ is positive constant, $0<\beta<\min \left\{\psi_{0}, m\right\}$. Suppose that there exists $\delta>0$ such that each connected component of the set

$D_{\delta}=V_{c} \cap\left\{x \in X: \frac{\left\|\Psi\left(\left[L_{g_{0}} V(x)\right]^{\mathrm{T}}\right)\right\|^{2}}{1+[\|\partial V(x) / \partial x\| \rho(x)]^{2}} \leq \delta\right\}$

is compact. Let the inclusion (17) be valid. Then along any solution of closed-loop system (6), (19) with the initial condition $x_{0}$ from the set $V_{c}$ the limit relation (3) holds.

See the proof of Theorem 8 in the appendix.

Remark 9. Theorems 2, 4 and 8 remain true when $k=n$ and the goal function $V(x)$ is positive definite: $V(x)>0$ $\forall x \neq 0, V(0)=0$. For this special case the set stabilization problem is obviously reduced to the stabilization of the equilibrium $x=0$ (see Lin, 1995, 1996). However, simple examples (see Example 2 in the next section) show that the new aforementioned theorems allow to establish the global (local) asymptotic stabilization even if the known stabilization results are not applicable. Another difference compared to the results of Lin $(1995,1996)$ is that Theorems 2 and 4 provide more general body of stabilizing regulators. Finally, it is worth mentioning that there is no analogue for Theorem 8 in Lin $(1995,1996)$.

\section{Examples}

In this section two examples illustrating the main results are presented.

\subsection{Example 1}

Consider the controlled pendulum, where the control action changes the length of the pendulum. The system equations are as follows:

$\left\{\begin{array}{l}\dot{p}=-\frac{a}{l+u} \sin q, \\ \dot{q}=p .\end{array}\right.$

Here $p \in R^{1}, q \in S^{1} ; u$ is a control variable; $a, l$ are some positive constants. The total energy of the unforced (with $u=0$ ) system (21) is

$H_{0}(q, p)=\frac{1}{2} p^{2}+\frac{a}{l}(1-\cos q)$.

Let $H_{*}$ be any positive constant. The problem is to design feedback control such that the limit relation

$\lim _{t \rightarrow+\infty}\left[H_{0}(q(t), p(t))-H_{*}\right]=0$

holds along solutions of the closed-loop system $(q(t), p(t))$. It is worth mentioning that such a problem could be considered as a stabilization of a prescribed rotational 
mode of the unforced pendulum. Introduce the functions

$V(q, p)=\frac{1}{2}\left[H_{0}(q, p)-H_{*}\right]^{2}$,

$y(q, p)=\frac{a}{l^{2}} p \sin q\left[H_{0}(q, p)-H_{*}\right]$.

For the sake of simplicity let us assume that $l>1$. Transform the system (21) into an equivalent form

$\dot{p}=-\frac{a}{l} \sin q+\frac{a}{l^{2}} \sin q u+R(q, p, u) u^{2}$,

$\dot{q}=p$,

where $R(q, p, u)=-a \sin q / l^{2}(l+u)$. One can easily check the validity of assumptions (A1) and (A2) and that

$\max _{\|u\| \leq 1}\|R(q, p, u)\| \leq \frac{a}{l^{2}(l+1)}$.

Thus inequality (11) holds with $\rho$ being the constant $a / l^{2}(l+1)$. Now let us define the set $\hat{\Omega}$, see (10). For this example $\operatorname{span}\{y(q, p)\} \subset S(q, p) \subset R^{1}$. Thus the set $P$, see (9), is contained in the union of the sets: $\{(q, p): p=0 ; q=0, \pi\},\left\{(q, p): H_{0}(q, p)=H_{*}\right\}$. Both sets are invariant with respect to the motions of the unforced pendulum and correspond to the downward and upright equilibria of the pendulum and the desired energy level. One can easily check that $P$ and $\hat{\Omega}$ coincide with the union of these sets. Let

$c= \begin{cases}\min \{V(\pi, 0), V(0,0)\} & \text { if } H_{*} \neq 2 a / l, \\ V(0,0) & \text { if } H_{*}=2 a / l .\end{cases}$

Such choice of $c$ implies that the set $\widehat{\Omega}_{c}$, see (16), contains only the desired energy level. Then Theorem 4 leads to the following statement.

Theorem 10. Consider the controlled pendulum (21). Take any regulator defined by relations (13) and (14), where

$\hat{\alpha}(q, p)=\frac{\beta}{1+\left[a / l^{2}(l+1)\right]^{2}\left[p^{2}+((a / l) \sin q)^{2}\right]}$.

Then along any solution $[q(t), p(t)]$ of the closed-loop system with initial conditions $[q(0), p(0)] \in V_{c}$ the goal relation (22) holds.

\subsection{Example 2}

To emphasize the difference between Theorems 2, 4 and the known related results (see Lin, 1995, 1996 Theorem 2.1; Theorems 3.2, 3.4, 3.8). Let us consider the following example: Suppose the system of interest has the form

$\dot{p}=-q+u$,

$\dot{q}=p$, where $q, p \in R^{1}$ and $u$ is control variable. Given the storage function $V \in C^{1}$, suppose

$V(q, p)=V_{1}(q)+V_{2}(p)$

and

$$
\begin{gathered}
V_{1}(q)>0, \forall q \neq 0, V_{2}(p)>0, \forall p \neq 0, \\
V_{1}(0)=V_{2}(0)=\dot{V}_{2}(0)=0 .
\end{gathered}
$$

One can easily check that system (23) together with the output

$y=\dot{V}_{2}(p)$

is passive with the storage function $V$. Consider the feedback control (see the relation (23) in Lin, 1996),

$u=-\frac{y}{1+y^{2}}$.

By Theorem 3.2 (Lin, 1996) the closed-loop system (23)-(25) is globally asymptotically stable if $S \cap \Omega=$ $\{(0,0)\}$ or $S \cap \widetilde{\Omega}=\{(0,0)\}$, where the sets $S, \Omega$ and $\widetilde{\Omega}$ are defined by relations (16), (17) in Lin (1996) (see also relations (5)-(7) in Lin (1995)). One can easily check that for the system (23)-(25), $\widetilde{\Omega}=R^{2}$ and

$S=\Omega=\left\{(q, p): \dot{V}_{2}(p)=0\right\}$.

Thus, the set $S \cap \Omega$ or $S \cap \widetilde{\Omega}$ contains at least the subset $\{(q, p): p=0\}$ and do not coincide with the equilibrium $(q, p)=(0,0)$. Therefore, Theorem 3.2 (Lin, 1996) does not guarantee the global asymptotic stability of the system (23)-(25). The same arguments show that Theorems 2.1 and 2.3 of Lin (1995) or Theorems 3.4 and 3.8 of Lin (1996) cannot be applied for the stability analysis of the system (23)-(25).

Let us check the conditions provided by Theorem 2 . One can easily check that

$P=\left\{(q, p): \dot{V}_{2}(p)=0\right\}$,

where the set $P$ is defined by (9). Due to $C^{1}$-smoothness and the positive definiteness of $V_{2}$ there exists $\varepsilon>0$ such that $\dot{V}_{2}(p) \neq 0$ for $0<\|p\|<\varepsilon$. This implies that the set $P$ does not contain a strip $\{(q, p): 0<\|p\|<\varepsilon\}$. In turn, this implies that the set $\hat{\Omega}$, see (10), consists only of the point $(0,0)$. Indeed, any solution of the unforced (with $u=0$ ) system (23) is a circle with constant radius. Any such circle with non-zero radius has nonempty intersection with the set $\{(q, p): 0<\|p\|<\varepsilon\}$. Thus, all the assumptions of Theorem 2 hold. It guarantees the global asymptotic stability of the closed-loop system (23)-(25).

\section{Appendix. Proof of Theorem 8}

Take any point $x_{0} \in V_{c}$ and consider the solution $x(t)=x\left(t, x_{0}\right)$ of the closed-loop system (1), (13). Similar to the proof of Theorem 3.3 of Shiriaev (2000a), we obtain 
that the solution $x(t)$ is well defined on $\left[0, t_{*}\right)$ with some $t_{*}>0$ and that for any $t \in\left[0, t_{*}\right)$ along this solution the inequality

$V(x(t))-V\left(x_{0}\right) \leq-C \int_{0}^{t} \alpha(x(\tau))|z(x(\tau))|^{2} \mathrm{~d} \tau$

holds. Here $z(x)=\Psi\left(\left[L_{g_{0}} V(x)\right]^{\mathrm{T}}\right), C=\left(\psi_{0}-\beta\right)>0$.

Let us show that $x(t)$ is well defined on $[0,+\infty)$. Indeed, it is obvious that $0<\alpha(x)<1$ and $0<\|u(x)\| \leq 1, \forall x$. The solution $x(t)$ is defined by the equation

$\dot{x}=f(x, u(x))$.

By the assumption the function $f(x, u)$ is bounded on the set $\Lambda=V_{c} \times\{u:|u| \leq 1\}$ and inequality (A.1) implies that the solution $x(t)$ belongs to $\Lambda$ whenever it is well defined. Hence we conclude that $|x(t)|$ grows not faster than a linear function, and, therefore, $x(t)$ is well defined on $[0,+\infty)$. In particular, we also conclude that inequality (A.1) is valid for $\forall t \geq 0$, and that the value of the integral

$\int_{0}^{+\infty} \alpha(x(\tau))|z(x(\tau))|^{2} \mathrm{~d} \tau$

is finite. Calculating the derivative of the function $\alpha(x)|z(x)|^{2}$ along the trajectory $x(t)$, we have

$\left.\frac{\mathrm{d}}{\mathrm{d} t}\left(\alpha(x)|z(x)|^{2}\right)\right|_{x=x(t)}=|z|^{2} \frac{\partial \alpha}{\partial x} f(x, u(x))+2 \alpha z^{\mathrm{T}} \frac{\partial z}{\partial x} f(x, u(x))$.

By the assumptions the right-hand side of (A.3) is uniformly bounded for any $t \in[0,+\infty)$. Therefore, by the Barbalat's lemma we derive from (A.2) that

$\lim \alpha(x(t))|z(x(t))|^{2}=0$.

$t \rightarrow+\infty$

The last limit relation implies that

$\lim _{t \rightarrow+\infty} \frac{\left\|\Psi\left(\left[L_{g_{0}} V(x(t))\right]^{\mathrm{T}}\right)\right\|^{2}}{1+[\|\partial V(x(t)) / \partial x\| \rho(x(t))]^{2}}=0$.

By the assumption the trajectory $x(t)$ belongs to one of the compact connected component of the set $D_{\delta}$, see (20). Thus, the trajectory $x(t)$ possesses non-empty compact $\omega$-limit set. To finish the proof we again invoke the arguments of Theorem 3.3 of Shiriaev (2000a), where the case of compact nonempty $\omega$-limit sets for any closedloop system solutions is treated.

\section{Conclusions}

In this paper, global and local stabilization of invariant sets for general nonlinear controlled systems is studied. It is assumed that a desired attractive set can be described by a zero value set of some smooth nonnegative scalar function. The main contribution of the paper are new sufficient conditions and the description of state feedback controllers that provide asymptotic stability of the goal set with specified region of attraction. The proofs are based on detailed analysis of the $\omega$-limit sets of the closed-loop system, using speed-gradient controllers for "affinized" controlled systems combined together with the $V$-detectability concept (Shiriaev, 2000a,b).

It is shown by example that even in the special case where the desired attractor of the controlled system consists of one equilibrium point (zero-state stabilization problem), the proposed conditions make possible to establish global (local) asymptotic stabilizability when other known conditions fail.

\section{References}

Åstrom, K. J., \& Furuta, K. (1996). Swinging up a pendulum by energy control. Proceedings of the 13th IFAC world congress, Vol. E (pp. 37-42).

Bloch, A. M., Krishnaprasad, P. S., Marsden, J. E., \& Sánchez de Alvarez, G. (1992). Stabilization of rigid body dynamics by internal and external torques. Automatica, 28, 745-756.

Byrnes, C. I., Isidori, A., \& Willems, J. C. (1991). Passivity, feedback equivalence and the global stabilization of minimum phase nonlinear systems. IEEE Transactions on Automatic Control, 36(11), $1228-1240$.

Chernousko, F. L., Akulenko, L. D., \& Sokolov, B. N. (1980). Control of oscillations. Moscow: Nauka (in Russian).

Fradkov, A. L. (1979). Speed-gradient scheme and its applications in adaptive control. Automation and Remote Control, 40(9), 1333-1342.

Fradkov, A. L. (1990). Adaptive control in complex systems. Moscow: Nauka (in Russian).

Fradkov, A. L. (1994). Nonlinear adaptive control: regulation-tracking-oscillation. Proceedings of first IFAC workshop on new trends in design of control systems, Smolenice (pp. 426-431).

Fradkov, A. L., Guzenko, P. Yu., Hill, D. J., \& Pogromsky, A. Y. (1995). Speed-gradient control and passivity of nonlinear oscillations. Proceedings of the third IFAC symposium on nonlinear control systems, Tahoe-City, USA, Vol. 2, pp. 655-659.

Fradkov, A. L. (1996). Swinging control of nonlinear oscillations. International Journal of Control, 64(6), 1189-1202.

Fradkov, A. L., Makarov, I. A., Shiriaev, A. S., \& Tomchina, O. P. (1997). Control of oscillations in Hamiltonian systems. Proceedings of fourth European control conference, Brussels, Paper No. 328.

Fradkov, A. L., \& Pogromsky, A. Yu. (1998). Introduction to control of oscillations and chaos. Singapore: World Scientific.

Fradkov, A. L., Miroshnik, I. V., \& Nikiforov, V. O. (1999). Nonlinear and adaptive control of complex systems. Dordrecht: Kluwer.

Hartman, Ph. (1964). Ordinary differetial equations. New York: Wiley.

Jurdjevic, V., \& Quinn, J. P. (1978). Controllability and stability. Journal of Differential Equations, 28, 381-389.

Lin, W. (1995). Feedback stabilization of general nonlinear control systems: A passive system approach. Systems \& Control Letters, 25 , 41-52.

Lin, W. (1996). Global asymptotic stabilization of general nonlinear systems with stable free dynamics via passivity and bounded feedback. Automatica, 32(6), 915-924.

Mahony, R., Mareels, I. M. Y., Campion, G., \& Bastin, G. (1997). Output stabilization of square non-linear systems. Automatica, 33(8), 1571-1577. 
Rumyantsev, V. V. (1970). On the optimal stabilization of controlled systems. Journal of Applied Mathematics \& Mechanics, 34(3), 415-430.

Shiriaev, A. S. (2000a). The notion of $V$-detectability and stabilization of invariant sets of nonlinear systems. Systems \& Control Letters, 39(5) 327-338.

Shiriaev, A. S. (2000b). Stabilization of compact sets for passive affine nonlinear systems. Automatica, to appear.

Sontag, Ed. (1981). Conditions for abstract nonlinear regulation. Information \& Control, 51, 105-127.

Vorotnikov, V. I. (1993). Partial stability and stabilization of motion: research approaches, results, distinctive characteristics. Automation and Remote Control, 54(3), 3-62.

Vorotnikov, V. I. (1998). Partial stability and control. Basal: Birkhäuser. Wiklund, M., Kristenson, A., \& Åström, K. J., (1993). A new strategy for swinging up an inverted pendulum. In Proceedings of the 12th IFAC world congress, vol. 9 (pp. 151-154).

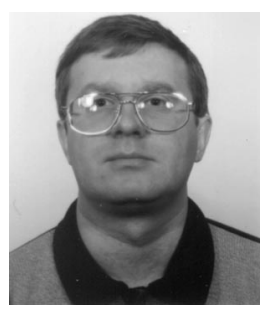

Anton S. Shiriaev was born in Kirov, Russia, in 1970. He received M.S. and Ph.D. degrees from the St. Petersburg State University, Russia, in 1993 and 1997 respectively, both in Applied Mathematics. He was a research fellow at the Institute for Problems of Mechanical Engineering, Russian Academy of Sciences, St. Petersburg in 1997 and a postdoctoral fellow at the Norwegian Institute of Science and Technology from February 1998 to December 1999. Since January 2000 he is appointed as an Assistant Professor at the Maersk Mc-Kinney Moller Institute for Production Technology, University of Southern Denmark.

His research interests in systems and control theory are related to stability and stabilization of nonlinear systems with emphasis to frequency domain theory and to systems with conserved quantities. He has also been interested in special linear quadratic optimization prob- lems such as existence of linear optimal 'universal' regulators when a system is subjected to either external harmonic disturbances with known frequencies and unknown amplitudes or singular stochastic disturbances with known upper bound for spectral densities.

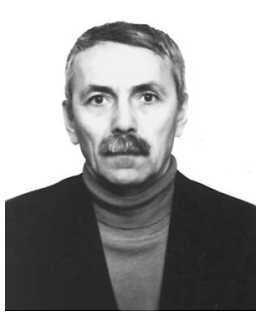

Alexander L. Fradkov was born on May 22, 1948; received the Diploma degree in mathematics from the Faculty of Mathematics and Mechanics of St. Petersburg State University (Department of Theoretical Cybernetics) in 1971; Candidate of Sciences (Ph.D.) degree in Engineering $\mathrm{Cy}-$ bernetics from St. Petersburg Mechanical Institute in 1975 and Doctor of Sciences degree in Control Engineering in 1986 from St. Petersburg Electrotechnical Institute. From 1971 to 1987 he has occupied different research positions. Since 1990 he has been the Head of the Laboratory of Complex Systems Control of the Institute for the Problems of Mechanical Engineering of Russian Academy of Sciences. He is also a part time Professor of St. Petersburg State University (Department of Theoretical Cybernetics).

His research interests are in fields of nonlinear and adaptive control, control of oscillatory and chaotic systems and computer-aided control systems design with applications to mechanical systems. He has published 13 books and textbooks. He is coauthor of 270 journal and conference papers and 9 patents.

Dr. Fradkov is the Vice-President of the St. Petersburg Informatics and Control Society, Member of the Russian National Committee of Automatic Control, IEEE Senior Member. Dr. Fradkov was Co-Chairman of 1-8 International Baltic Student Olympiades on Automatic Control in 1991-2000; NOC Chairman of the First and Second International IEEE-IUTAM Conference "Control of Oscillations and Chaos" in 1997 and 2000; associate editor of European Journal of Control; a member of IEEE Control Systems Society Conference Editorial Board. He was an official representative of Russia at the IFAC General Assembly during 14the IFAC World Congress in 1999. 